\title{
Study on the adsorption of heavy metals by biochar modified with different modifiers under room temperature
}

\author{
Jiajia Ding ${ }^{1,2 *}$, Yu Tian ${ }^{1}$, Tao Lu ${ }^{1}$ and Jiang Kang ${ }^{1}$ \\ ${ }^{1}$ College of Geography and Environmental Engineering, Lanzhou City University, Lanzhou 730070, PR China \\ ${ }^{2}$ Gansu Mining Area Pollution Control and Ecological Restoration Engineering Research Center
}

\begin{abstract}
In order to obtain an environment-friendly biochar adsorption material with excellent adsorption performance, the biochar made from corn straw was modified with $\mathrm{FeCl}_{3}, \mathrm{Na}_{2} \mathrm{~S}$ and $\mathrm{KMnO}_{4}$ as modifiers, and the adsorption effect of biochar on several heavy metal ions in water before and after modification was studied. The properties of biochar before and after modification were characterized by SEM, XRD, FTIR and BET, and the adsorption mechanism was discussed. The results showed that the three modified reagents could effectively improve the adsorption capacity of biochar for three heavy metal ions in water, and the adsorption effect was still good at room temperature. The adsorption difference is related to the specific surface area, pore structure, aromatic structure and $\mathrm{SiO}_{2}$ content of biochar. The adsorption mechanism mainly includes the complexation reaction between physical adsorption and surface functional groups. This study is beneficial to promote the resource utilization of agricultural and forestry waste biomass, and provides a certain reference basis for related research.
\end{abstract}

\section{Introduction}

Rapid economic and social development has brought about a large number of heavy metal pollutants, which seriously harm the ecological environment and biological health ${ }^{[1]}$. Biochar $(\mathrm{BC})$ is a kind of porous carbon material with wide sources, environmental protection and low $\operatorname{cost}^{[2]}$. Due to its unique high aromatic and heterocyclic structure, complex pore structure, large specific surface area, stable structural capacity and abundant surface functional groups, it has good adsorption capacity and can effectively repair heavy metal pollution ${ }^{[3]}$. However, the current heavy metal pollution, especially the heavy metal pollution in the soil is complex and diverse, and the original $\mathrm{BC}$ cannot achieve the ideal adsorption effect. The adsorption performance of $\mathrm{BC}$ can be improved by modification treatment, such as increasing specific surface area, pore complexity or changing the functional group substance category on the surface, so as to achieve better treatment effect of heavy metals. The chemical modification methods are widely used, including acid modification, alkali modification, oxidant modification, metal oxide modification and carbonaceous material modification $^{[4]}$. For example, acid modification can remove metals and other impurities on the surface of $\mathrm{BC}$, and introduce acidic functional group substances ${ }^{[5]}$. Alkaline modification can increase the surface area of BC and introduce oxygen-containing functional groups ${ }^{[6-7]}$. Oxidants can also introduce oxygen-containing functional groups, activate the surface functional groups of $\mathrm{BC}$ and increase their active $\operatorname{sites}^{[8]}$. In this study, ferric chloride
$\left(\mathrm{FeCl}_{3}\right)$, sodium sulfide $\left(\mathrm{Na}_{2} \mathrm{~S}\right)$ and potassium permanganate $\left(\mathrm{KMnO}_{4}\right)$ were selected as modifiers to study the adsorption of three heavy metals by biochar before and after modification under room temperature. The purpose of this study is to compare the modification effect and mechanism of different kinds of modified reagents on biochar at room temperature, so as to provide a basis for subsequent practical application. Although the specific surface area of biochar from corn straw is not the optimal choice, as agricultural waste, it has a large yield and is of great significance for its comprehensive utilization.

\section{Materials and methods}

\subsection{Biochar modification}

Corn stalk biochar was purchased from Suzhou City, Jiangsu Province. It was pre-dried to a constant weight and then screened with a diameter of $0.425 \mathrm{~mm}$ before utilization. The modified biochar with the best adsorption effect on the three kinds of heavy metal ions was screened out at room temperature $\left(20^{\circ} \mathrm{C}\right)$ through the preexperiment, and the optimal modification concentration of the corresponding modified reagent was obtained. The optimal concentration of the selected modifier was $0.4 \mathrm{~mol} / \mathrm{L}$ of $\mathrm{FeCl}_{3}$ solution, $0.4 \mathrm{~mol} / \mathrm{L}$ of $\mathrm{Na}_{2} \mathrm{~S}$ solution and $0.2 \mathrm{~mol} / \mathrm{L}$ of $\mathrm{KMnO}_{4}$ solution. The original biochar is marked as $\mathrm{BC}$, the modification of $\mathrm{FeCl}_{3}$ is marked as $\mathrm{BC}$ $\mathrm{F}$, the modification of $\mathrm{Na}_{2} \mathrm{~S}$ is marked as BC-S, and the modification of $\mathrm{KMnO}_{4}$ is marked as BC-P. The above

\footnotetext{
* Corresponding author: chrisding0202@163.com
} 
three kinds of modified biochar and BC were used as raw materials for subsequent adsorption experiments.

\subsection{Characterizations}

Scanning electron microscope (SEM) images of biochar before and after modification were obtained with a JSM6701F Scanning Microscope. Their X-ray diffraction (XRD) measurements were performed within the angle range $2 \theta=5-90^{\circ}$ using a Shimazu X-ray diffractometer XRD-6100 type. Fourier transform infrared spectra (FTIR) for the samples were generated by a PerkinElmer spectrum 3 FT-IR Spectrometer $\left(400-4,000 \mathrm{~cm}^{-1}\right)$. The BET surface area, pore surface, and pore volume of these samples were performed on the Feldman ASAP 2020 physical adsorption instrument.

\subsection{Adsorption experiment}

The adsorption properties of three kinds of modified biochar on $\mathrm{Cr}^{6+}, \mathrm{Pb}^{2+}$ and $\mathrm{Cu}^{2+}$ were determined by batch experiments. Every result came from at least three parallel samples. The initial concentration of heavy metal solution was $50 \mathrm{mg} / \mathrm{L}$, with the vibration rate was $120 \mathrm{r} / \mathrm{min}$. All tests were carried out at room temperature $\left(20^{\circ} \mathrm{C}\right)$. The three influencing factors of solution $\mathrm{pH}$, adsorption time and biochar dosage were studied. The concentrations of $\mathrm{Cr}^{6+}, \mathrm{Pb}^{2+}$ and $\mathrm{Cu}^{2+}$ in water samples were determined by spectrophotometry. The Langmuir adsorption mode ${ }^{[9]}$ and Freundlich adsorption model ${ }^{[10-11]}$, were used to simulate the experimental data.

\section{Results and discussion}

\subsection{Initial assessment}

Table1. Adsorption capacity of three kinds of heavy metals before and after modification of biochar.

\begin{tabular}{|c|c|c|c|}
\hline $\begin{array}{c}\text { The } \\
\text { adsorption } \\
\text { quantity } \\
\text { (mg/g) }\end{array}$ & $\mathbf{C r}^{6+}$ & $\mathbf{P b}^{\mathbf{2 +}}$ & $\mathbf{C u}^{2+}$ \\
\hline BC & 11.58 & 20.55 & 16.25 \\
\hline BC-F & 29.76 & 24.39 & 23.92 \\
\hline BC-S & 29.57 & 24.47 & 23.49 \\
\hline BC-P & 29.57 & 24.86 & 23.66 \\
\hline
\end{tabular}

As is shown in Table 1, the modified biochar still has a good adsorption effect at room temperature. When the biochar is modified by $\mathrm{FeCl}_{3}$ or $\mathrm{Na}_{2} \mathrm{~S}$, the optimal concentration of the modifier is $0.4 \mathrm{~mol} / \mathrm{L}$. the optimal concentration of $\mathrm{KMnO}_{4}$ modification is $0.2 \mathrm{~mol} / \mathrm{L}$, which may be because the adsorption promotion effect of oxidation on biochar can only be realized at relatively low concentration. The modified biochar with the best adsorption effect on $\mathrm{Cr}^{6+}$ and $\mathrm{Cu}^{2+}$ was $\mathrm{BC}-\mathrm{F}$, and the best adsorption effect on $\mathrm{Pb}^{2+}$ was BC-P.

\subsection{SEM analysis}

As can be seen from the SEM image in Fig.1., BC has a relatively smooth surface, which is in the shape of sheets of different sizes, and the pores are not obvious. After modification, its surface has undergone significant changes. The apparent characteristics of the three kinds of modified biochar are not exactly the same. Generally speaking, the surface pores increase significantly, and tend to be regular, and the specific surface area was enlarged by the pore broadening effect, which strengthens the adsorption site on the surface of biochar itself and improves the adsorption capacity ${ }^{[12]}$. The pore structure of $\mathrm{BC}-\mathrm{F}$ is the most regular, and the size of the flake particles of $\mathrm{BC}-\mathrm{S}$ is uniform. The surface of BC-P is the roughest, which may be the result of oxidation.
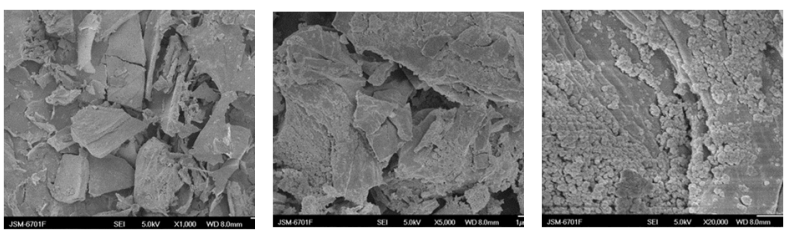

BC
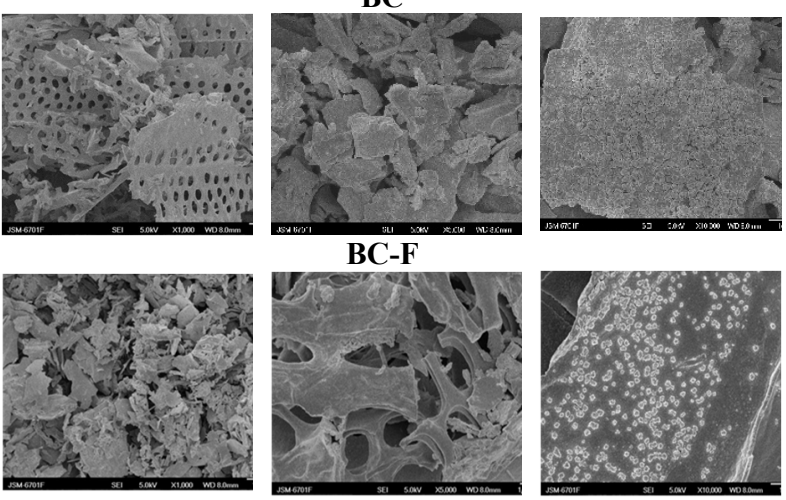

BC-S
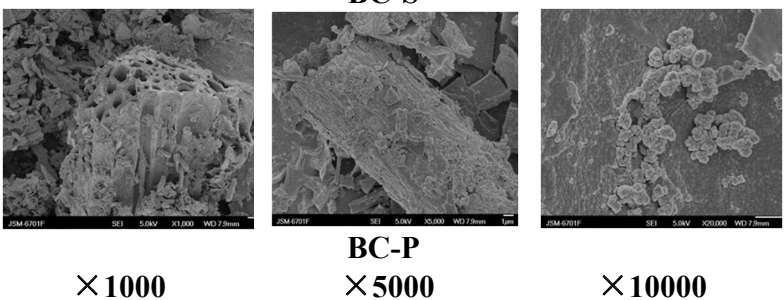

$\times 1000$

$\times \mathbf{1 0 0 0 0}$

Figure 1. SEM images of biochar before and after modification

\subsection{XRD analysis}

As shown in Fig.2., compared with BC, the crystal phase structure of $\mathrm{BC}-\mathrm{F}$ and $\mathrm{BC}-\mathrm{S}$ did not change greatly, indicating that the use of $\mathrm{FeCl}_{3}$ and $\mathrm{Na}_{2} \mathrm{~S}$ to modify biochar did not affect the crystal structure. The crystal structure of BC-P is destroyed due to the strong oxidation of $\mathrm{KMnO}_{4}$. The crystal surface diffraction peaks of the modified biochar at $2 \theta=26^{\circ}$ and $40^{\circ}$ are obviously weakened. BC-F and BC-S showed new characteristic peaks at $16.6^{\circ}$ and $76.6^{\circ}$, indicating that the corresponding groups were introduced. 


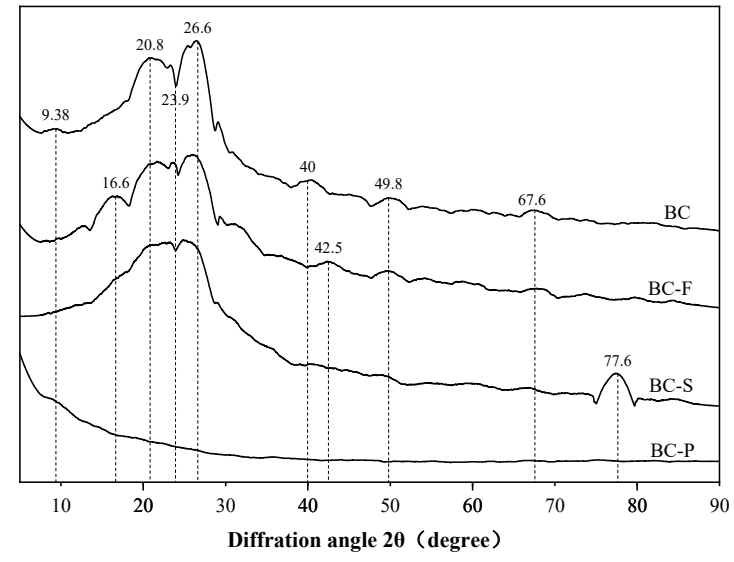

Figure 2. XRD analysis of biochar before and after modification

\subsection{FT-IR spectra analysis}

As can be seen from the FTIR spectra in Fig.3., the wide peak at 3391 3405 $\mathrm{cm}^{-1}$ was the stretching vibration of hydroxyl O-H. The absorption peak at $2915 \sim 2921 \mathrm{~cm}^{-1}$ is methylene asymmetric stretching vibration. The carbonyl $(\mathrm{C}=\mathrm{O})$ had a peak at around $1585 \sim 1597 \mathrm{~cm}^{-1}[11,13]$. The carbon oxygen bonds $(\mathrm{C}-\mathrm{O}, \mathrm{C}-\mathrm{O}-\mathrm{C})$ had a peak at around $1059 \sim 1076 \mathrm{~cm}^{-1}$, and carboxyl $(-\mathrm{COOH})$ had a peak near $1378 \mathrm{~cm}^{-1}[11,14]$. The peak at $1378 \mathrm{~cm}^{-1}$ was markedly increased in BC-S spectra after adsorption, this peak was the result of metal carboxylate $(-\mathrm{COO})^{[11,15]}$, which proved that metal ions interacted with organic functional groups during adsorption process. The absorption peaks at
1434 and $1075 \mathrm{~cm}^{-1}$ are C-O stretching vibration and $\mathrm{O}-\mathrm{H}$ in-plane bending vibration absorption peaks, and the peak at $875 \mathrm{~cm}^{-1}$ is $\mathrm{C}-\mathrm{H}$ in-plane bending vibration in the aromatic ring. Compared with the $\mathrm{BC}$, the position of absorption peak and the intensity change in the infrared absorption spectrum did not obvious.

\subsection{BET analysis}

After modification, the specific surface area and pore volume of biomass carbon increased significantly, while the average pore size decreased. The larger the specific surface area was, the more adsorption sites could be provided, so that the adsorption effect was significantly improved (Table 1). The porous structures of biochar before and after modification were quantitatively characterized by $\mathrm{N}_{2}$ adsorption method. According to the adsorption-desorption isotherm (Fig.4.), it can be seen that the modification process has a great influence on the formation of biomass carbon pore structure. The original biochar samples were close to II type isotherm, but not typical, indicating that the pore size structure was large and the distribution was irregular. The modified biochar samples showed a typical IV isotherm, which indicated that the samples were mesoporous structure, and the modification reagent led to the coalescence of pores, leading to the formation of mesoporous structure. The hysteresis loop in the isotherm is between HL type and H3 type, indicating that although the modified biochar still belongs to the slit pore characteristics formed by polymer sheet particles, the pore structure tends to be evenly distributed, and the adsorption capacity is significantly improved.

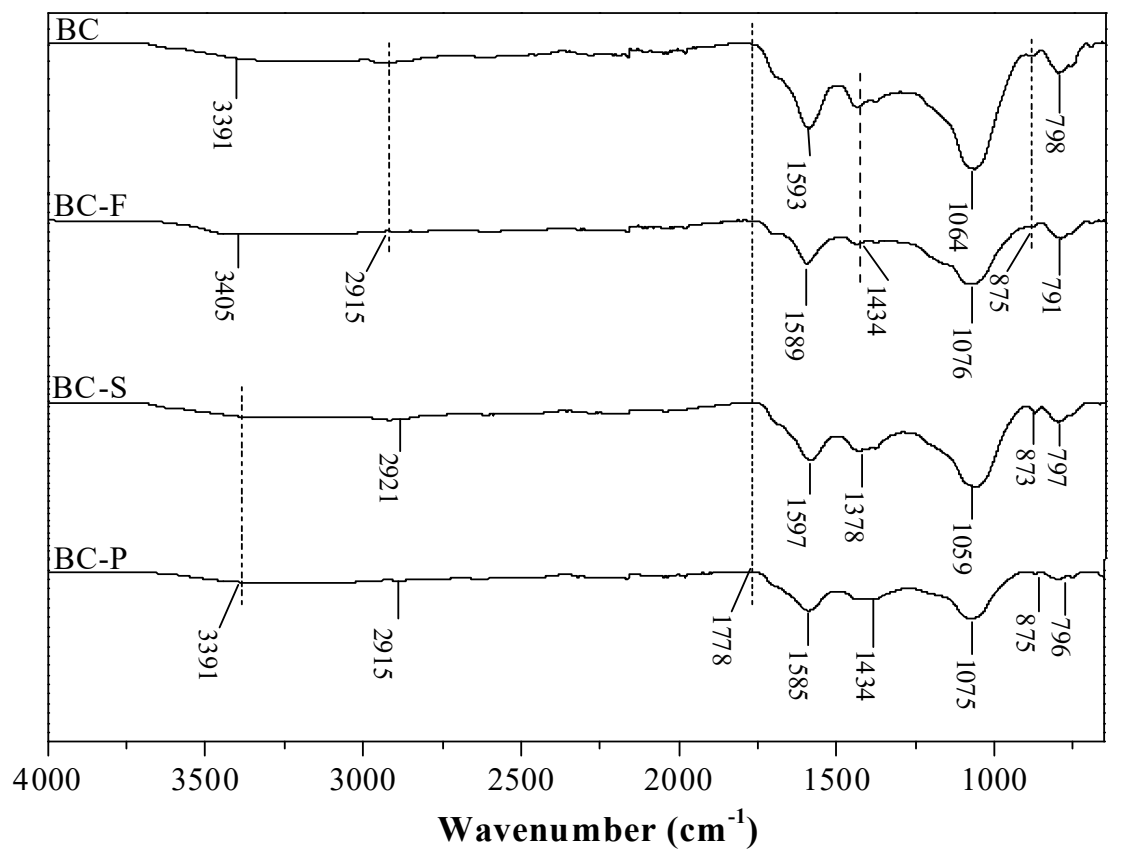

Figure 3. FT-IR spectra of biochar before and after modification 
Table2. Surface area and pore volume of biochar before and after modification

\begin{tabular}{|c|c|c|c|c|c|c|}
\hline Product ID & $\begin{array}{c}\text { BET specific } \\
\text { surface } \\
\left(\mathrm{m}^{3} / \mathrm{g}\right)\end{array}$ & $\begin{array}{c}\text { Micropore } \\
\text { surface areas } \\
\quad\left(\mathrm{m}^{3} / \mathrm{g}\right)\end{array}$ & $\begin{array}{c}\text { External } \\
\text { specific } \\
\text { surface } \\
\text { areas }\left(\mathrm{m}^{2} / \mathrm{g}\right) \\
\end{array}$ & $\begin{array}{c}\text { Total pore } \\
\text { volume } \\
\left(\mathrm{cm}^{3} / \mathrm{g}\right)\end{array}$ & $\begin{array}{l}\text { Micropore } \\
\text { volumes } \\
\left(\mathrm{cm}^{3} / \mathrm{g}\right)\end{array}$ & $\begin{array}{c}\text { Average } \\
\text { pore } \\
\text { width } \\
(\mathrm{nm}) \\
\end{array}$ \\
\hline $\mathrm{BC}$ & 0.1760 & 5.5563 & -5.3803 & 0.0048 & 0.0027 & 101.9823 \\
\hline BC-F & 5.4575 & 1.3405 & 4.1170 & 0.0115 & 0.0008 & 10.0261 \\
\hline BC-S & 11.0621 & 0.8222 & 10.2400 & 0.0214 & 0.0005 & 8.1276 \\
\hline BC-P & 3.9397 & - & 4.9679 & 0.0131 & -0.0151 & 16.1592 \\
\hline
\end{tabular}
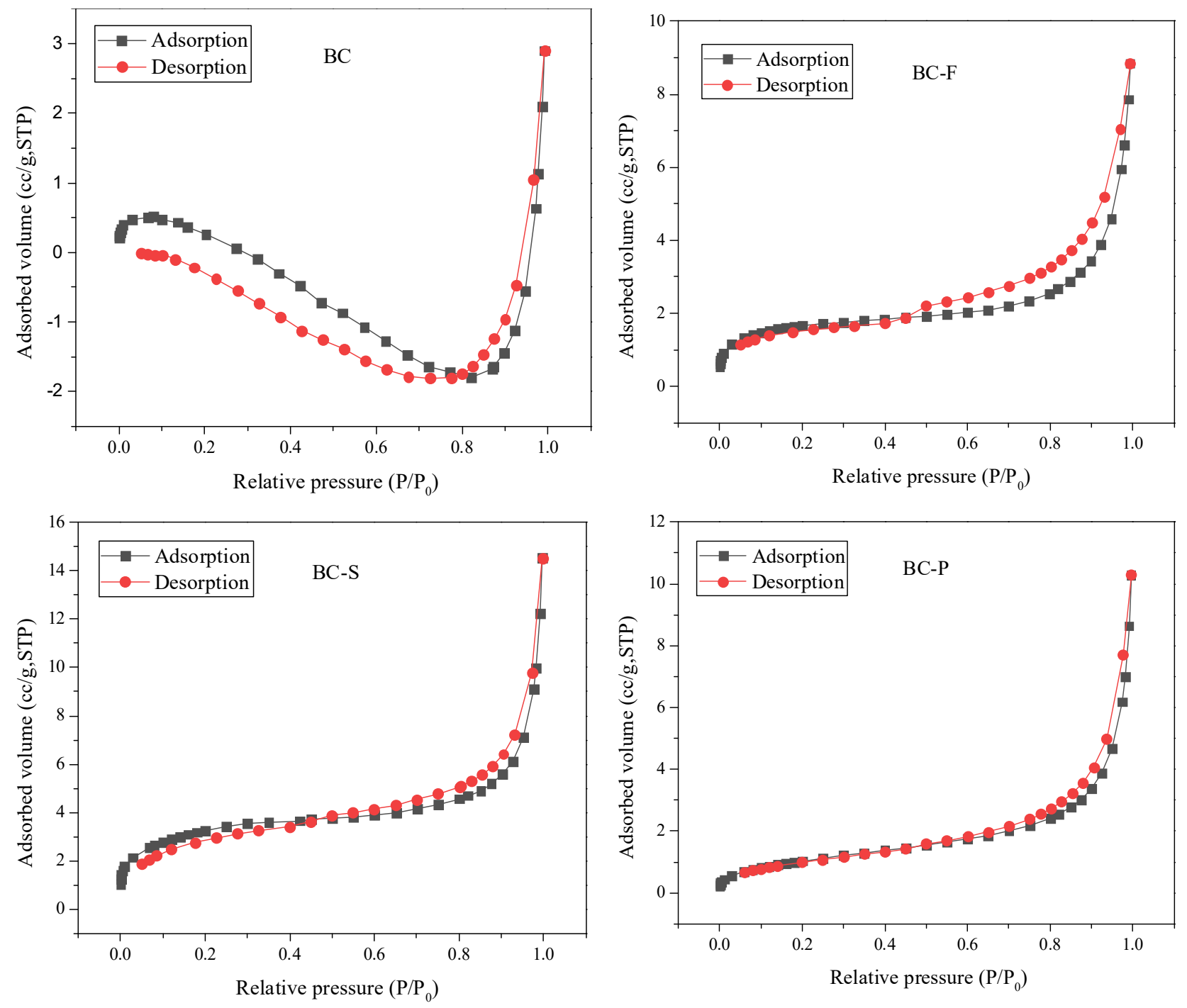

Figure 4. BET analysis of biochar before and after modification

\section{Conclusions and perspectives}

The results show that the biochar modified by $\mathrm{FeCl}_{3}, \mathrm{Na}_{2} \mathrm{~S}$ and $\mathrm{KMnO}_{4}$ has a good adsorption effect on heavy metals of $\mathrm{Cd}, \mathrm{Pb}$ and $\mathrm{Cu}$ at room temperature. As an oxidizing modifier, $\mathrm{KMnO}_{4}$ can only promote the adsorption of biochar at a relatively low modified concentration. The modified biochar with the best adsorption effect on $\mathrm{Cr}^{6+}$ and $\mathrm{Cu}^{2+}$ was $\mathrm{BC}-\mathrm{F}$, and the best adsorption effect on $\mathrm{Pb}^{2+}$ was $\mathrm{BC}-\mathrm{P}$. At room temperature, the $\mathrm{pH}$ of the solution is the main factor affecting the adsorption, and the optimal removal $\mathrm{pH}$ of each metal is different. 
After modification, surface pores of the biochar increase significantly, and tend to be regular, and the specific surface area was enlarged by the pore broadening effect. There are still some differences in the apparent morphology of the three different modified biochar. The XRD analysis showed that the content of $\mathrm{SiO}_{2}$ decreased after modification, and among the three modifiers, only $\mathrm{KMnO}_{4}$ would cause the destruction of the crystal structure of biochar. The infrared spectrum analysis showed that the surface functional groups of the prepared biochar had no significant change before and after modification, mainly containing hydrophilic functional groups such as carboxyl group and hydroxyl group as well as aromatic ring structure. The difference of adsorption effect of biochar before and after biochar comes from the difference of specific surface area, pore structure, aromatic structure and $\mathrm{SiO}_{2}$ content. That is, the larger the specific surface area, the richer the pore structure, the more adsorption sites provided, and the stronger the electrostatic adsorption capacity of heavy metals. The higher the degree of aromatization, the stronger the cationic $\pi$ bond. In order to further explore the mechanism, more experimental studies and analysis are needed.

\section{Acknowledgments}

This work was partly supported by Lanzhou City University Doctoral Research Initiation Fund (LZCUBS2018-05). The authors also thank the anonymous reviewers for their invaluable insight and helpful suggestions.

\section{References}

1. J.X. Li, L.C. Wu, J. Zhang, C.S. Wang, Q.Q. Yu, Y. Peng, Y.H. Ma, Ecol. Environ. Sci, 24(2015)

2. H. Dong, J. Deng, Y. Xie, C. Zhang, Z. Jiang, Y. Cheng, K. Hou, G. Zeng, J. Hazard. Materi., (2017)

3. X. Zhou, J. Zhou, Y. Liu, Fuel, 233(2018)

4. J.H. Luo, Y.J. Gao, T.H. Song, Y. Chen, Water Sci. Technol., (2021)

5. A.U. Rajapaksha, S.S. Chen, Tsang, C. W. Daniel, M. Zhang, M. Vithanage, S. Mandal, B. Gao, N.S. Bolan, Y.S. Ok, Chemosphere, 148 (2016)

6. Z. Ding, X. Hu, Y. Wan, S. Wang, B. Gao, J. Ind. Eng. Chem., 33 (2016)

7. M.E Doumer, A. Rigol, M. Vidal, Mangrich, Environ. Sci. Pollut. Res., 23(2016)

8. Y.C.Li, J.G.Shao, X.H.Wang, Energy Fuels, 28(2014)

9. Langmuir, J. Chem. Phys., 40(2015)

10. C. Ng, J.N. Losso, W.E. Marshall, R.M. Rao, Bioresour. Technol., 85(2002)

11. X.L. Hu, Y.W. Xue, L.N. Liu, Y.F. Zeng, L. Long, Environ. Sci. Pollut. Res., (2018)

12. H.X.Wang, J.L. Xu, L.X. Sheng, J. Cleaner Prod, 2020

13. S. Yorgun, D. Yıldız, J Taiwan Inst Chem Eng, 53(2015)
14. V. Frišták, E. Víglašová, L. Ďuriška, M. Galamboš, E. Moreno-Jimenéz, M. Pipíška, G. Soja, J. Iran. Chem. Soc., 14(2017)

15. A.H.M. Ibrahim, A. Jalbout, Appl. Spectrosc, 62(2008) 